\title{
Reconstruction of Structured Quadratic Pencils from Eigenvalues on Ellipses and Parabolas
}

\author{
R. Ibragimov, V. Vatchev* \\ Department of Mathematics, University of Texas at Brownsville \\ One University Boulevard, Brownsville,TX 78575,USA
}

\begin{abstract}
In the present paper we study the reconstruction of a structured quadratic pencil from eigenvalues distributed on ellipses or parabolas. A quadratic pencil is a square matrix polynomial

$$
Q P(\lambda)=M \lambda^{2}+C \lambda+K,
$$

where $M, C$, and $K$ are real square matrices. The approach developed in the paper is based on the theory of orthogonal polynomials on the real line. The results can be applied to more general distribution of eigenvalues. The problem with added single eigenvector is also briefly discussed. As an illustration of the reconstruction method, the eigenvalue problem on linearized stability of certain class of stationary exact solution of the Navier-Stokes equations describing atmospheric flows on a spherical surface is reformulated as a simple mass-spring system by means of this method.
\end{abstract}

Keywords and phrases: structured quadratic pencil, inverse problems, complex eigenvalues, orthogonal polynomials

Mathematics Subject Classification: 65F18, 15A22, 42C05

\section{Introduction}

Recently a serious effort is concentrated in studying inverse problems where one is interested in deriving system's characteristics from known data, for an overview see [3]. The goal of the present paper is to study the reconstruction of a special structured quadratic pencil from given complex eigenformations. A quadratic pencil is the square matrix polynomial

$$
Q P(\lambda)=M \lambda^{2}+C \lambda+K
$$

for a real singular variable $\lambda$ and real square matrices $M, C$, and $K$. One of the areas where these polynomial matrices arise is the mathematical modelling of serially linked mass-spring systems. The simplest system is shown on Figure 1.

\footnotetext{
${ }^{*}$ Corresponding author. E-mail: vesselin.vatchev@utb.edu
} 


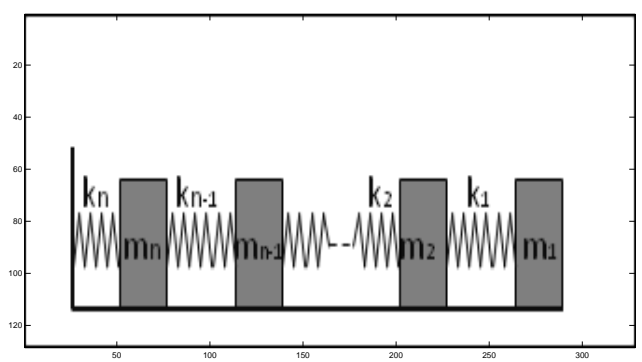

Figure 1

The masses $m_{1}, \ldots, m_{n}$ are serially linked by springs with positive spring constants $k_{1}, \ldots, k_{n}$. The whole system is connected to a stationary base by the spring $k_{n}$. If damping is considered then a set of real positive numbers $c_{1}, \ldots, c_{n}$ represents the damping constants associated to each of the masses, for more details see [3]. The problem can be formulated for other physical systems including a vibrating beam, a composite pendulum, or serially linked electric circuits.

For a mass-spring system consisting of $n$ masses the matrix $M$ is diagonal with positive entries $\left(m_{1}, m_{2}, \ldots, m_{n}\right)$. The types of connections determine the structures of $C$ and $K$. A typical matrix $K$, usually referred to as a stiffness matrix, corresponding to the springs connecting the masses, has the following structure

$$
\left(\begin{array}{cccc}
\sum_{j=1}^{n} k_{1, j} & -k_{1,2} & & -k_{1, n} \\
-k_{2,1} & \sum_{j=1}^{n} k_{2, j} & & -k_{2, n} \\
& & \ddots & \\
-k_{n, 1} & -k_{n, 2} & & \sum_{j=1}^{n} k_{n, j}
\end{array}\right)
$$

where $k_{i, j}=k_{j, i} \geq 0$ is the spring constant of the spring connecting the $i$-th and the $j$-th masses. If there is no connection between the $i$-th and the $j$-th masses then $k_{i, j}=k_{j, i}=0$. The entry $k_{i, i}$ is the spring constant of the spring connecting the $i-$ th mass to the base. Similarly, the entries of $C$ are defined depending on the damping conditions.

In the paper we study systems with limited connectivity, namely each mass is connected to no more than $s$ of its neighboring masses in each direction, i.e. $c_{i, j}=k_{i, j}=0$ if $|i-j|>s$. That restriction corresponds to band-limited matrices $K$ and $C$. Due to the lack of connections to the base for masses with indices $i<n-s$ we impose the conditions that the sums of the elements in the rows corresponding to these masses equals 0 . The types of matrices $K$ and $C$ used in the paper are introduced in the next definition.

Definition 1.1. A real matrix $L=\left(l_{i, j}\right)_{i, j=1}^{n}$ is a $s$-connectivity matrix if it is symmetric, with band length $2 s+1$ i.e. $l_{i, j}=0$ for $|i-j|>s$, and $\sum_{i=1}^{n} l_{i, j}=0, i=1, \ldots, n-s$.

If in addition all of the entries on the main diagonal are positive and all of the entries off the main diagonal are non-positive we refer to $L$ as a $s$-connectivity stiffness matrix.

The eigenvalues of the quadratic pencil (1.1) are the solutions of the equation $\operatorname{det} Q P(\lambda)=0$. For any eigenvalue $\lambda_{j}$, an eigenvector $y_{j}$ is defined as a solution of the linear system $Q P\left(\lambda_{j}\right) y_{j}=0$.

The problem to construct a pencil $Q P(\lambda)$ from eigenformations is known as Inverse Eigenvalue Problem. Extensive study of that problem with non structured $K$ and $C$ or pure complex eigenvalues is done in numerous papers, we mention just a few: [2] - [5]. In the case when the eigenvalues are purely imaginary the matrix $C$ is the zero matrix. The problem of reconstructing pencil $M \lambda^{2}+K$ with a tri-diagonal matrix $K$ ( 1-connectivity matrix) is solved in [2], [4], and [5]. The case of purely imaginary eigenvalues corresponds to modeling a mass-spring system with no damping. A standard approach for solving that 
problem is to consider the eigenvalues as zeros of the $n$-th polynomial and the matrix $K$ as the Jacobi matrix of a system of orthogonal polynomials on the real line. That approach as, well as, direct solutions by studying the structure of the matrix $K$ can be found in [4]. The case of eigenvalues with non-zero real and imaginary parts corresponds to a system of orthogonal polynomials on a curve in the plane. The recurrence relations there are of different kind and do not allow direct connection to the Inverse Eigenvalue Problem. The case of non-zero real parts corresponds to damping and the author is not aware of any constructive work in that direction. In the current paper we consider an approach that benefits from the theory of the orthogonal polynomials on the real line. By considering special characterization of the complex eigenvalues we obtain necessary conditions on the distribution of the eigenvalues such that the matrices $K$ and $C$ are $s$ - connectivity stiffness matrices. Explicit constructions and physical interpretations for $s \leq 2$ are discussed. The set of pencils with prescribed eigenvalues in general depends on certain number of parameters. In some cases it is important the reconstructed pencil to have a prescribed eigenvector. We consider adding an eigenvector to the initial information in Remark 7.

In Section 2 we give brief overview of some of the main properties of orthogonal polynomials on the real line and obtain the characterization of special type of structured pencils having prescribed eigenvalues. We establish necessary conditions for connectivity type matrices i.e. band-limited with the particular sign distributions of the entries as in Definition 1. In section 3 we study the structure of the pencil depending on the distribution of the eigenvalues $\lambda$ 's in the complex plane. Particular case, studied in details, is when all of the eigenvalues belong to an ellipse. The type of problems studied in the existing literature deal with eigenvalues on vertical lines in the complex plane, and hence the results in the paper are natural extensions of those results.

\section{Reconstruction of Structured Quadratic Pencil from Eigenvalues}

The problem of serially linked masses with no damping, Figure 1, is well studied and understood. The construction of the corresponding pencil can be done by using orthogonal polynomials on the real line, see [3]. Band-limited matrices are natural objects in the study of orthogonal polynomials and the approach that we consider in the current section uses extensively the theory of orthogonal polynomials on the real line.

For an inner product $\langle r, t\rangle$, by using the Gram-Schmidt orthogonalization to $1, x, x^{2}, \ldots$, we obtain a system of orthonormal polynomials $p_{k}, k=0,1, \ldots$ The degree of $p_{k}$ is exactly $k$ and $\left\langle p_{i}, p_{j}\right\rangle=\delta_{i, j}$, where $\delta_{i, j}=1$ if $i=j$ and 0 otherwise. The theory of the orthogonal polynomials on the real line is studied in great details. For completeness we list some properties, that can be found for example in [9], used extensively in the current section. A characteristic property of the orthogonal polynomials is that they satisfy a three-term recurrence relation $p_{-1}=0, p_{0}=1$, and $x p_{k}(x)=c_{k+1} p_{k+1}(x)+b_{k} p_{k}(x)+$ $c_{k} p_{k-1}(x), c_{k}>0$ for $k=1,2, \ldots$ The zeros $z_{j}^{k}$ of $p_{k}$, for $j=1, \ldots, k$, are all simple and real, and there is at least one zero of $p_{k-1}$ between any two consecutive zeros of $p_{k}$. The last property is known as the interlacing property of the zeros.

If the inner product is supported on a negative set (interval, half-line, or infinite or finite discrete set), then from the interlacing property of the zeros it follows that $p_{k}(0) \neq 0$, and hence the polynomials $q_{k}(x)=$ $p_{k}(x) / p_{k}(0)$ are well defined. The scaled orthogonal polynomials $q_{k}$ satisfy a three-term recurrence relation of the form

$$
x q_{k}(x)=a_{k+1} q_{k+1}(x)-\left(a_{k+1}+a_{k}\right) q_{k}(x)+a_{k} q_{k-1}(x),
$$

with $q_{-1}(x)=0, a_{0}=0$, and positive numbers $a_{k}, k \geq 1$. The Jacobi matrix for the first $n$ polynomials $q_{0}, \ldots, q_{n-1}$ is defined as

$$
J:=\left(\begin{array}{cccccc}
-a_{1} & a_{1} & 0 & \ldots & 0 & 0 \\
a_{1} & -\left(a_{1}+a_{2}\right) & a_{2} & \ldots & 0 & 0 \\
& & \ddots & & & \\
0 & 0 & 0 & \ldots & a_{n-1}-\left(a_{n-1}+a_{n}\right)
\end{array}\right) .
$$


It is clear that $-J$ is 1 -connectivity stiffness matrix. Let $\Re^{n}$ be the Euclidean $n$-dimensional space and $D_{\gamma}$ denote the diagonal matrix with entries the components of the vector $\gamma=\left(\gamma_{1}, \ldots, \gamma_{n}\right) \in \Re^{n}$. If $z_{n}<z_{n-1}<\ldots<z_{1}<0$ are the zeros of $p_{n}$ then, it is well known( see [9]) that the matrix

$$
U=\left(q_{i-1}\left(z_{j}\right)\right)_{i, j=1}^{n}=\left(\begin{array}{ccccc}
q_{0}\left(z_{1}\right) & \ldots & q_{0}\left(z_{j}\right) & \ldots & q_{0}\left(z_{n}\right) \\
& & \ddots & & \\
q_{i-1}\left(z_{1}\right) & \ldots & q_{i-1}\left(z_{j}\right) & \ldots & q_{i-1}\left(z_{n}\right) \\
& \ddots & & \\
q_{n-1}\left(z_{1}\right) & \ldots & q_{n-1}\left(z_{j}\right) & \ldots & q_{n-1}\left(z_{n}\right)
\end{array}\right)
$$

is non-singular and the columns of $U$ form an orthogonal basis of $\Re^{n}$. The inverse matrix is $U^{-1}=$ $\left(\gamma_{i} q_{j-1}\left(z_{i}\right) p_{j-1}^{2}(0)\right)_{i, j=1}^{n}$. Very useful formula relating $U$ and $J$ is $U D_{\lambda} U^{-1}=J$ (see [4], [9]). The conjugation of an arbitrary diagonal $D_{\mu}$ matrix with the matrix $U$ can be expressed by using the Gram matrix of the polynomials $q$. Indeed, let $\mu \in \Re^{n}$ and $M(z)$ be the unique interpolating polynomial $M\left(z_{j}\right)=\mu_{j}, j=1, \ldots, n$, of the least possible algebraic degree. Let the Gram matrix for the polynomials $q_{0}, \ldots, q_{n-1}$ associate with $M$ be the symmetric matrix

$$
G(M)=\left(\begin{array}{ccccc}
\left\langle q_{0}, q_{0} M\right\rangle & \ldots & \left\langle q_{0}, q_{j} M\right\rangle & \ldots & \left\langle q_{0}, q_{n-1} M\right\rangle \\
\left\langle q_{i-1}, q_{0} M\right\rangle & \ldots & \left\langle q_{i-1}, q_{j} M\right\rangle & \ldots & \left\langle q_{i-1}, q_{n-1} M\right\rangle \\
\left\langle q_{n-1}, q_{0} M\right\rangle & \ldots & \left\langle q_{n-1}, q_{j} M\right\rangle & \ldots & \left\langle q_{n-1}, q_{n-1} M\right\rangle
\end{array}\right) .
$$

Let $p^{2}(0)=\left(p_{0}^{2}(0), \ldots, p_{n-1}^{2}(0)\right), D_{0}=D_{p^{2}(0)}, D_{0}^{-1}$ be the inverse of $D_{0}$, and $D_{1}$ be the identity matrix. Since $\left\langle q_{i}, q_{i}\right\rangle=1 / p_{i}^{2}(0)$ then we get that

$$
U D_{\mu} U^{-1}=\left(p_{j}^{2}(0)\left\langle q_{i}, q_{j} M\right\rangle\right)_{i, j=0}^{n-1}=G(M) D_{0}, \quad G(M)=U D_{\mu} U^{-1} D_{0}^{-1} .
$$

Since $M$ is algebraic polynomial of degree not exceeding $n-1$, it follows that $G(M)=\sum_{s=0}^{n-1} d_{s} G\left(x^{s}\right)$, for some real $d$ 's. Let $r_{s}(x)=x^{s}, s=0,1, \ldots, n-1$ then the following lemma holds true.

Lemma 2.1. The matrix $G\left(x^{s}\right)$ is a s-connectivity matrix for $s=1, \ldots, n-1$ and $G(1)$ is diagonal.

Proof. It is clear that for any $s$ the matrix $G\left(r_{s}\right)$ is symmetric. Since $q$ is orthogonal set, then $\left\langle q_{i}, q_{j} r_{s}\right\rangle=$ 0 if $|i-j|>s$. The sum of the entries in the $k$-th row equals $\left\langle\sum_{i=0}^{n-1} q_{i}, q_{k} r_{s}\right\rangle$. For $s=0$ the sum is $\left\langle\sum_{i=0}^{n-1} q_{i}, q_{k}\right\rangle=1 / p_{k}^{2}(0)$, and the lemma is proven in that case. For $s>0$ we use induction to prove that $\left\langle\sum_{i=0}^{n-1} q_{i}, q_{k} r_{s}\right\rangle=0$ for $k \leq n-s$. For $s=1$ using the recurrence relation $r_{1} q_{k}=a_{k+1} q_{k+1}-\left(a_{k+1}+\right.$ $\left.a_{k}\right) q_{k}+a_{k} q_{k-1}$ for $k=1, \ldots, n-2$ we get

$$
\begin{aligned}
\left\langle\sum_{i=0}^{n-1} q_{i}, q_{k} r_{1}\right\rangle & =a_{k+1}\left\langle\sum_{i=0}^{n-1} q_{i}, q_{k+1}\right\rangle \\
& -\left(a_{k+1}+a_{k}\right)\left\langle\sum_{i=0}^{n-1} q_{i}, q_{k}\right\rangle_{\gamma, z}+a_{k}\left\langle\sum_{i=0}^{n-1} q_{i}, q_{k-1}\right\rangle=0 .
\end{aligned}
$$

For $k=n-1$ the sum is $\left\langle\sum_{i=0}^{n-1} q_{i}, q_{n} r_{1}\right\rangle=-a_{n} \neq 0$. For transparency we also consider the case $s=2$. The recurrence for $q$ implies that $r_{2} q_{k}=r_{1}\left(a_{k+1} q_{k+1}-\left(a_{k+1}+a_{k}\right) q_{k}+a_{k} q_{k-1}\right)$ and for $k<n-2$ the case $s=1$ provides row sums 0 . For $k=n-2$ we get $\left\langle\sum_{i=0}^{n-1} q_{i}, q_{n-2} r_{2}\right\rangle=-a_{n} a_{n-1} \neq 0$, and for 
$k=n-1$ we get $\left\langle\sum_{i=0}^{n-1} q_{i}, q_{n-1} r_{2}\right\rangle=-a_{n}\left(a_{n}+a_{n-1}\right) \neq 0$. Assuming that the statement is true for $s$, the statement for $s+1$ follows from the recurrence $r_{s+1} q_{k}=r_{s} r_{1} q_{k}$. The proof is complete.

From the above lemma and the comments preceding it, it follows that the conjugation of a diagonal matrix with the matrix $U$ results in a connectivity type matrix up to a right matrix multiplication by a diagonal matrix. Next we use that observation to construct structured quadratic pencils. By $i$ we denote the imaginary unit.

Lemma 2.2. Let $\lambda_{j}=\alpha_{j}+i \beta_{j}, j=1, \ldots, n$ be given complex numbers, $\left\{p_{k}\right\}_{k=0}^{\infty}$ be a system of orthogonal polynomials, and $z_{n}<z_{n-1}, \ldots<z_{1}$ be the zeros of $p_{n}$. Let $A(z)$ and $B(z)$ be the interpolating polynomials $A\left(z_{j}\right)=-2 \alpha_{j}$ and $B\left(z_{j}\right)=\alpha_{j}^{2}+\beta_{j}^{2}$, and $G(A)$ and $G(B)$ be the corresponding Gram matrices. Then the structured quadratic pencil

$$
D_{0}^{-1} \lambda^{2}+G(A) \lambda+G(B)
$$

has eigenvalues $\lambda_{j}, j=1, \ldots, n$.

Proof. By using (2.1) the determinant of $D_{0}^{-1} \lambda^{2}+G(A) \lambda+G(B)$ is equal to

$$
\begin{array}{r}
\operatorname{det}\left(D_{0}^{-1} \lambda^{2}+G(A) \lambda+G(B)\right)=\operatorname{det}\left(D_{1} \lambda^{2}+G(A) D_{0} \lambda+G(B) D_{0}\right) \operatorname{det}\left(D_{0}^{-1}\right) \\
=\operatorname{det}(U) \operatorname{det}\left(D_{1} \lambda^{2}+D_{\alpha} \lambda+D_{\beta}\right) \operatorname{det}\left(U^{-1}\right) \operatorname{det}\left(D_{0}^{-1}\right)=\prod_{j=1}^{n} \frac{\lambda^{2}-2 \alpha_{j} \lambda+\alpha_{j}^{2}+\beta_{j}^{2}}{p_{j}^{2}(0)}
\end{array}
$$

and the identity $\lambda^{2}-2 \alpha_{j} \lambda+\alpha_{j}^{2}+\beta_{j}^{2}=\left(\lambda-\lambda_{j}\right)\left(\lambda-\bar{\lambda}_{j}\right), \bar{\lambda}_{j}=\alpha_{j}-i \beta_{j}$ completes the proof.

Remark 2.3. From the proof of Lemma 3, it follows that the quadratic pencil $D_{1} \lambda^{2}+D_{\alpha} \lambda+D_{\beta}$ has eigenvalues $\lambda_{j}, j=1, \ldots, n$, but the matrices $D_{\alpha}$ and $D_{\beta}$, that correspond to $C$ and $K$, have non-zero sums of the row elements. From physical point of view this means that each mass is connected to the base and not to any of the other masses.

The problem for the distribution of the signs of the entries of $G(A)$ and $G(B)$ is more delicate. We establish necessary conditions depending on the linearization coefficients of orthogonal polynomials. For a system of orthogonal polynomials $\left\{p_{k}\right\}_{k=0}^{\infty}$ the product $p_{k} p_{j}$ is a polynomial of degree $k+j$, and hence can be expressed as a linear combination of the polynomials $p_{m}, m=0, \ldots, k+j$ in the following form

$$
p_{k} p_{j}=\sum_{m=|k-j|}^{k+j} c(m, k, j) p_{m} .
$$

The coefficients $c(m, k, j)$ are called linearization coefficients and the process of finding them is known as linearization of orthogonal polynomials. An important question, studied by Askey [1], Gasper [6], Szwarc [8], and others, is to determine when they are non-negative. There are many algorithms [8] to calculate the linearization coefficients and many criteria to establish their non-negativeness depending on the recurrence coefficients, which is quite useful in the case since the polynomials $p_{k}$ are obtained through the Gram-Schmidt process.

We provide a necessary condition on the distribution of the eigenvalues, such that $C$ and $K$ are connectivity stiffness matrices, if all $c(m, k, j)$ are non-negative and a second necessary condition if $c(m, k, j)$ are arbitrary but the inner product is a discrete and finite. The latter amounts to finding positive solutions of linear systems of inequalities which is a basic topic in Linear Programming. 
Theorem 2.4. Let $\lambda_{j}=\alpha_{j}+i \beta_{j}, \alpha_{j}<0$ be $n$ complex conjugated numbers. If $\left\{p_{k}\right\}_{k=0}^{\infty}$ is a sequence of orthogonal polynomials for an inner product supported on a negative set $I, c(m, k, j)$ are the linearization coefficients, and $z_{j}, j=1, \ldots, n$ are the zeros of $p_{n}$, then the matrices $C$ and $K$ are connectivity stiffness matrices if one of the following two holds:

I.) All $c(m, k, j)$ are positive. The polynomials $A$ and $B$, defined in Lemma 3, are positive on $I$, have the representations $A(x)=d_{0}-\sum_{j=1}^{n-1} d_{j} p_{j}(x)$ and $B(x)=e_{0}-\sum_{j=1}^{n-1} e_{j} p_{j}(x)$ for some $d_{j}>0, e_{j}>0, j=1, \ldots, n-1$, and $A(0)=0, B(0)=0$;

II.) The inner product is $\sum_{j=1}^{n} \gamma_{j} f\left(z_{j}\right) g\left(z_{j}\right), \gamma_{j} \geq 0$ and the system of linear inequalities

$$
\begin{array}{r}
\sum_{j=1}^{n} \gamma_{j} c(m, k, j) q_{k}\left(z_{j}\right) \mu_{j}<0, \\
\mu_{j}>0
\end{array}
$$

for $k=2,3, \ldots, n-1$ is satisfied in the case $\mu_{j}=-2 \alpha_{j}$ and in the case $\mu_{j}=\alpha_{j}^{2}+\beta_{j}^{2}$.

Proof. It is enough to show that the matrix $C$ is a connectivity stiffness matrix. For $K$ the proof is identical. First we prove I. Since $A$ is a polynomial of degree no larger than $n-1$ it has a representation $A(x)=\sum_{m=0}^{n-1} d_{m}^{\prime} p_{m}(x)$. From Lemma 1, it follows that in order $C$ to be a connectivity type matrix it is necessary that $A(0)=0$. Furthermore, the entries of $G(A)$ are

$$
\left\langle A, q_{k} q_{m}\right\rangle=\frac{1}{p_{k}^{2}(0) p_{m}^{2}(0)}\left\langle A, p_{k} p_{m}\right\rangle=\sum_{j=|k-m|}^{n-1} \frac{c(m, k, j)}{p_{k}^{2}(0) p_{m}^{2}(0)} d_{j}^{\prime} .
$$

Since all $c$ 's are positive it follows that the entries of $G(A)$ off the main diagonal, i.e. $k \neq m$ are negative if $d_{j}^{\prime}<0, j=1,2, \ldots, n-1$. The entries on the main diagonal, i.e. $k=m$, are $\left\langle A, q_{k} q_{k}\right\rangle$ and thus they are positive if $A$ is a positive polynomial on $I$. This ends the prove of I.

II is trivial. If $\mu_{j}=-2 \alpha_{j}>0$ or $\mu_{j}=\alpha_{j}^{2}+\beta_{j}^{2}>0$ we have the following sign restrictions on the entries of $G(A)$.

$$
\begin{array}{r}
\left\langle A, q_{k} q_{m}\right\rangle=\sum_{j=1}^{n} \gamma_{j} c(m, k, j) q_{k}\left(z_{j}\right) \mu_{j}<0, \\
\mu_{j}>0,
\end{array}
$$

for $k, m=1,2, \ldots, n-1$ and $k \neq m$. For the elements on the main diagonal, i.e. $k=m$, we have that $\left\langle A, q_{k} q_{k}\right\rangle=\sum_{j=1}^{n} \gamma_{j} \mu_{j} q_{k}^{2}\left(z_{j}\right)>0$. The proof is complete.

The systems of polynomials with non-negative linearization coefficients are well studied and characterized, for more information see [8] and the references within. We mention only, that for a system $\left\{p_{k}\right\}_{k=0}^{\infty}$ with non-negative linearization coefficients the change of variable $y=x-b$ does not change the signs of the linearization coefficients, and hence any polynomial sequence $q_{k}(x)$, such that $p_{k}(x)=q_{k}(x+b)$ has non-negative linearization coefficients, too. In [6] it is established that the linearization coefficients for the Jacobi polynomials are non-negative. Since they are defined on the interval $[-1,1]$, by changing the variable $y=x-1$, we obtain polynomials that satisfy all of the conditions in Theorem 1 . In [8] a necessary conditions on the monotonicity of the recursion coefficients are established.

The second condition in Theorem 1 is more direct and we use it in the next section to characterize pencils with eigenvalues belonging to ellipses. 


\section{Applications}

In the previous section we proved necessary conditions based on the knowledge of the zeros of orthogonal polynomials and properties of their linearization coefficients. For the localization of zeros and calculation of linearization coefficients there are well established effective numerical procedures, but very few explicit formulas are known. In this section we exploit the second condition in Theorem 1. For an inner product $\sum_{j=1}^{n} \gamma_{j} f\left(z_{j}\right) g\left(z_{j}\right), \gamma_{j} \geq 0$, supported on a finite and discrete set, it is clear that, the zeros of the $n-t h$ polynomial are $z_{j}, j=1, \ldots, n$.

For $\lambda_{j}=\alpha_{j}+i \beta_{j}, j=1, \ldots, n$ let $A$ and $B$ be as in Lemma 2. If $z_{n-j}=-\left(\alpha_{j}^{2}+\beta_{j}^{2}\right)$ then $K$ is the Jacobi matrix for $q$, which is 1 -connectivity stiffness matrix. From Theorem 1 , it follows that if $A(0)=0$ then the matrix $C$ is $s$-connectivity with $s$ being the degree of $A$. Three particular cases for $s \leq 2$ are discussed bellow.

In the case when $\lambda$ 's are on a vertical line, i.e. $\alpha_{j}=-c, c>0$, the polynomial $A$ is a constant, and hence $C$ is a diagonal matrix. The structured pencil in that case is $D_{0}^{-1} \lambda^{2}+2 c D_{1} \lambda-J$. For $c \neq 0$ the matrix $c D_{1}$ is not $s-$ connectivity. For $c=0$ we obtain a pencil with no damping and the corresponding mass-spring system is shown on Figure 1. This problem is solved using different techniques in [2], [3], and [4].

In [7], we studied linearized stability of incompressible viscous fluid flows in a thin spherical shell, using the two-dimensional Navier-Stokes (NS) equations on a sphere which ignore the effects of rotation. In particular, it has been shown in [7] that when the spherical layer is truncated between two symmetrical rings, the obtained stationary exact solutions of the NS equations remain asymptotically stable for all Reynolds number. The inquiry is motivated by dynamically significant Coriolis forces in oceanographic applications such as a climate variability models and the general atmospheric (or oceanic) circulation model.

As a particular application to the reconstruction of structured quadratic pencils discussed here, we will approximate the resulting eigenvalue problem in [7] by a simple mass-spring system; the latter approximation roots on the hypothesis that there is a quadratic relation between the real and imaginary parts of the corresponding eigenvalues of the problem that were found numerically in [7].

\subsection{Eigenvalues on an Ellipse}

In the case when $\lambda$ 's are on the circle centred at $(a, 0), a \neq 0$ and radius $R \leq|a|$ the polynomial $A(z)=c_{1} z+c_{0}$, with $c_{0}=\frac{R^{2}-a^{2}}{a}$, and $c_{1}=-\frac{1}{a}$. The matrix $C$ is three-diagonal and is 1 -connectivity stiffness matrix if, and only if, $a<0$ and $|a|=R$, i.e. $\alpha_{j}=c_{1} \beta_{j}$. In the particular case when $\lambda$ 's belong to the unit circle centred at $(-1,0)$ we have that $C=K=-J$. The mass-spring system corresponds to serially linked masses with the $k$-th mass connected to the $k-1$-st and $k+1$-st only.

The restriction $R \leq|a|$ excludes from consideration the unit circle centred at the origin. By using the previous result we can obtain a quadratic pencil in the case $\left|\lambda_{j}\right|=1$. Indeed, it is clear that $\mu_{j}=\lambda_{j}-1$ belongs to the unit circle centred at $(1,0)$, and hence is an eigenvalue of the pencil $D_{0}^{-1} \mu^{2}-J \mu-J$. It follows that $\lambda$ 's are eigenvalues of the pencil

$$
D_{0}^{-1}(\lambda+1)^{2}-J(\lambda+1)-J=D_{0}^{-1} \lambda^{2}+\left(2 D_{0}^{-1}-J\right) \lambda+D_{0}^{-1},
$$

which is equivalent to $D_{1} \lambda^{2}+\left(2 D_{1}-J D_{0}\right) \lambda+D_{1}$.

The case considered above is well studied and solved by using different techniques. In the case when the eigenvalues belong to an ellipse the author is not aware of any constructive work. Next theorem shows how the technique used in the previous section can be successfully applied to that problem under very mild and natural conditions on the lengths of the axes of the ellipse.

Theorem 3.1. Let $\lambda_{j}=\alpha_{j}+i \beta_{j}$ be $n$ complex conjugated numbers distributed on an ellipse $(\alpha+u)^{2}+\frac{\beta^{2}}{v^{2}}=$ $u^{2}, u>0, v>1$, and $\frac{2 u v^{2}}{v^{2}-1}>\max _{j}\left|\alpha_{j}\right|$. Then for the pencil obtained in Theorem 5, part II, with nodes 
$z_{n-j}=\sigma \alpha_{j}$, for any $\sigma>0$, the matrices $C$ and $K$ are correspondingly 1 and $2-$ connectivity stiffness matrices.

Proof. Fix $\sigma>0$ and consider the inner product $\langle f, g\rangle=\sum_{k=1}^{n} \gamma_{k} f\left(z_{k}\right) g\left(z_{k}\right)$ for an arbitrary positive vector $\left(\gamma_{1}, \ldots, \gamma_{n}\right)$. Then $C$ is the negative of the Jacobi matrix for the system of orthogonal polynomials with that inner product, and hence $1-$ connectivity stiffness matrix. If $\epsilon=\frac{v^{2}-1}{\sigma^{2}}>0$ and $T=\frac{2 u v^{2}}{v^{2}-1} \sigma>$ $\max _{j}\left|\alpha_{j}\right| \sigma$, then $B(z)=-\epsilon z(z+T)$ and $B\left(z_{j}\right)>0$, for $j=1, \ldots, n$. From Lemma 2 , it follows that $K=$ $G(B)$ is 2 - connectivity matrix. The entries on the main diagonal of $G(B)$ are $\sum_{k=1}^{n} \gamma_{k} B\left(z_{k}\right) q_{j}^{2}\left(z_{k}\right)>0$. For the elements on the second, from the main, diagonal we have that $\left\langle B, q_{j} q_{j+2}\right\rangle=-\frac{\epsilon}{p_{j}^{2}(0) p_{j+2}^{2}(0)} \frac{a_{j}}{a_{j+2}}<0$. Finally, since $\left\langle z, q_{j} q_{j+1}\right\rangle=\frac{a_{j+1}}{p_{j}^{2}(0) p_{j+1}^{2}(0)}>0$ and $z<-\sigma \max _{j}\left|\alpha_{j}\right|<0$, it follows that

$$
-\frac{\left\langle z^{2}, q_{j} q_{j+1}\right\rangle}{\left\langle z, q_{j} q_{j+1}\right\rangle} \leq \max |z|=\sigma \max _{j}\left|\alpha_{j}\right|<T
$$

and hence $\left\langle B, q_{j} q_{j+1}\right\rangle<0$. From the symmetry of $G(B)$ we complete the proof.

We conclude the case with a remark regarding a prescribed eigenvector.

Remark 3.2. The eigenvectors of a pencil obtained in Lemma 3 are the columns of $U$, and hence they are eigenvectors of the Jacobi matrix for the system $q$. The problem for constructing a pencil with prescribed either real or purely imaginary complex eigenvalues and a given eigenvector is well known inverse eigenvalue problem with no damping and is completely solved in [4]. In the setting consider in the paper a prescribed eigenvector imposes conditions on the weights $\gamma_{j}, j=1, \ldots, n$.

\subsection{Eigenvalues on a Parabola}

An experimental system of eigenvalues is finite and obtained with a certain precision. If the original system has infinitely many eigenvalues we can extrapolate them from the first few obtained. In order to use the technique from Section 2 we need to consider a system of orthogonal polynomials on an infinite interval. The classical polynomials on the real line include several families which zeros can be approximated numerically. In the example that follows on the interval $(-\infty, 0]$ we consider a modification of the Laugerre polynomials $L_{k}$ normalized by $L_{k}(0)=1$. The orthogonal polynomials with respect to the inner product

$$
\int_{-\infty}^{0} e^{x} P_{n}(x) P_{m}(x) d x=\int_{0}^{\infty} e^{-x} P_{n}(-x) P_{m}(-x) d x
$$

are $P_{k}(x)=L_{k}(-x)$. From the properties of the Laguerre polynomials by changing the variable $x=-x$ we obtain that $P_{k}(0)=1,\left\langle P_{k}, P_{j}\right\rangle=\delta_{k, j}$ and $P_{0}(x)=1, P_{1}(x)=x+1, P_{2}(x)=\frac{x^{2}}{2}+2 x+1$ and the recurrence relation

$$
x P_{k}(x)=-(k+1) P_{k+1}(x)+(2 k+1) P_{k}(x)-k P_{k-1}(x) .
$$

The zeros of $P_{k}$ are $-l_{j, k}, j=1, \ldots, k$ i.e. the zeros of $L_{k}$ multiplied by -1 .

In [7] the linearized stability of incompressible viscous fluid flows in a thin spherical shell was investigated by using two-dimensional Navier-Stokes equations on a sphere. It has been shown that the resulting eigenvalue problem can be written as the following coupled system of ODEs:

$$
L_{k} \Psi_{k}=\Phi_{k}, \quad L_{k} \Phi_{k}+\epsilon, \quad \Phi_{k}^{\prime}=\mu \Phi_{k} .
$$

In Eq. (3.1) the dependent and independent variables are defined as follows: $\Psi_{k}(\theta)$ is the stream function, $\theta$ is being latitude angle, $k$ is a wave number, $\Phi_{k}$ is defined as $\Phi_{k}=\Delta_{k} \Psi_{k}$, in which

$$
\Delta_{k}=\frac{d^{2}}{d \theta^{2}}+\cot \theta \frac{d}{d \theta}-\frac{k^{2}}{\sin ^{2} \theta},
$$




$$
L_{k}=\frac{d}{d x}\left[\left(1-x^{2}\right) \frac{d}{d x}\right]-\frac{k^{2}}{1-x^{2}}
$$

is the Sturm-Liouville operator for associated legendre functions, $\epsilon$ is the Reynolds number of the basic flow associated with the exact solution which stability is under the question and $\mu$ is the eigenvalue.

The system (3.1) is defined on the symmetric interval $\left[-x_{0}, x_{0}\right]$. where $x_{0}=\cos \theta_{0}$. The spherical layer $S_{0}$ corresponds to the case $0<x_{0}<1$, while the complete sphere $S$ corresponds to the limit $x_{0} \rightarrow 1$. In the latter case, the interval $[-1,1]$ connects two singular points $x= \pm 1$ of the Sturm-Liouville operator (3.3). For different values of the parameters $k$ and $\epsilon$ the first few eigenvalues has been numerically computed in [7]. Examining the plots we notice that there is a quadratic relation between the real and imaginary parts. In other words the eigenvalues for a fixed stream function $\mid p s i_{k}$ and $\epsilon$ are distributed on parabola. To check our hypothesis we fix $k=1, \epsilon=1$, and consider the zeros of $L_{21}$. The values of $l_{j, 21}$ in increasing order are:

$$
\begin{array}{r}
0.0673,0.3548,0.8737,1.6269,2.6186,3.8547,5.3424,7.0912 \\
9.1128,11.4218,14.0363,16.9790,20.2785,23.9718,28.1075 \\
32.7515,37.9972,43.9852,50.9474,59.3260,70.2557
\end{array}
$$

Next the real and the imaginary parts of the eigenvalues $\mu_{j}$ are approximated by the complex numbers $\nu_{j}=\alpha_{j}+i \beta_{j}, j=1, \ldots, 21$, where $\alpha_{j}=-a l_{j, 21}-b, a=\frac{56}{l_{21,21}}, b=35$ and $\beta_{j}=-\sqrt{c l_{21, j}-d}, c=$ $\frac{70^{2}}{l_{21,21}-0.75 l_{5,21}}, d=\frac{70^{2} 0.75 l_{5,21}}{l_{21,21}-0.75 l_{5,21}}$. In Figure $2 \mu$ are the circles and $\nu$ are the $x^{\prime}$ s.

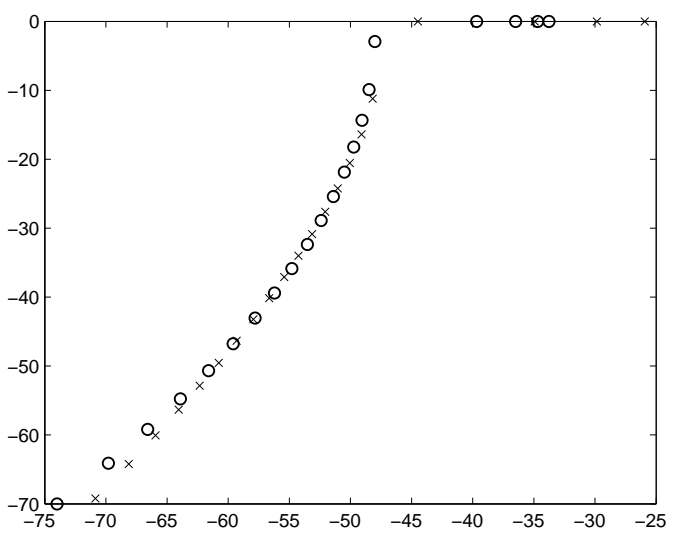

Figure 2

The approximation can be improved if the modified Laguerre polynomials are used but we see that even in the approximation considered above the set of $\nu$ 's exhibits the main characteristics of the system $\mu$. The first few eigenvalues are real and the rest are with imaginary parts distributed on a parabola $x=a_{0} y^{2}+c_{0}$ for some real $a_{0}, c_{0}$. From the numerical eigenvalues it follows that they are also distributed on parabolas for varying $k$ and $\epsilon$.

Considering the general problem for eigenvalues on a parabola we see that the corresponding interpolation polynomials are linear $A(x)=a x+b=(b-a) P_{0}(x)+a P_{1}(x), a>0, b>0$ and quadratic $B(x)=c x^{2}+d x+e=(e-d+2 c) P_{0}(x)+(d-4 c) P_{1}(x)+2 c P_{2}(x), c>0, d>0$. In the next lemma we construct a Structured Quadratic Pencils with eigenvalues $\nu_{j}$. 
Lemma 3.3. Let the complex numbers $\nu_{j}=\alpha_{j}+i \beta_{j}, j=1, \ldots$, n be such that $\alpha_{j}=-A\left(l_{j}\right) / 2, \alpha_{j}^{2}+\beta_{j}^{2}=$ $B\left(l_{j}\right)$, where $l_{j}$ are the zeros of $P_{n}$ then the quadratic pencil

$$
D_{1}^{-1} \lambda^{2}+\left(a J+b D_{1}\right) \lambda+e D_{1}+d J+c G\left(x^{2}\right),
$$

where $G\left(x^{2}\right)$ is the symmetric five diagonal matrix with $a_{j, j}=(j+1)^{2}+(2 j+1)^{2}+j^{2}, a_{j, j-1}=-4 j^{2}$, and $a_{j, j-2}=j(j-1)$ has eigenvalues $\nu_{j}$.

Proof. From the normalization $P_{j}(0)=1$ we have that $D_{0}=D_{1}$. Clearly $G(x)=J$ - Jacobian matrix of the polynomials $P$. For $G\left(x^{2}\right)$ we have that

$$
a_{m, j}=\left\langle P_{m} z, P_{j} z\right\rangle=\left\langle-(m+1) P_{m+1}+(2 m+1) P_{m}-m P_{m-1},-(j+1) P_{j+1}+(2 j+1) P_{j}-j P_{j-1}\right\rangle,
$$

and hence $a_{m, j}=0$ if $|m-j|>2$ and $a_{j, j}=(j+1)^{2}+(2 j+1)^{2}+j^{2}, a_{j-1, j}=a_{j, j-1}=-4 j^{2}, a_{j-2, j}=$ $a_{j, j-2}=j(j-1)$. The proof is complete.

We conclude the paper with few remarks about the stream function $\Psi_{k}$. The eigenvalues $\mu$ are obtained from the differential equation (3.1) which is linear non-constant coefficient second order. The quadratic pencil that we obtained is five diagonal and corresponds to the mass-spring system in Figure 3. All of the masses are 1 and connected by springs with increasing spring constants $k_{j}$ consecutively and the squares represent the damping of the systems. All of the even index masses are also connected consecutively as well as all of the odd index masses.

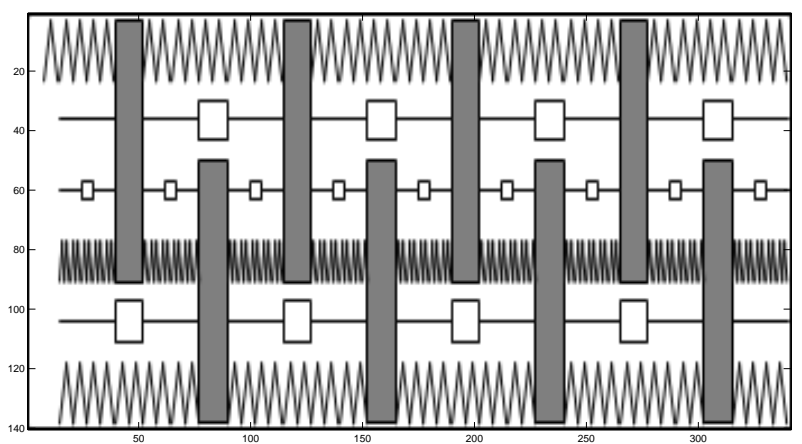

Figure 3

\section{References}

[1] R. Askey. Linearization of the product of orthogonal polynomials. Problems in Analysis (R. Gunning, ed.), Princeton University Press, Princeton, N.J., (1970), 223-228.

[2] Y. Cai, Y. Kuo, W. Lin, S. Xu. Solutions to a quadratic inverse eigenvalue problem. Linear Algebra Appl., 430 (2008), $1590-1606$.

[3] M. Chu, G. Golub. Structured inverse eigenvalue problems? Acta Numer., 11 (2002), 1-71.

[4] M. Chu, Y. Kuo, W. Lin. On inverse quadratic eigenvalue problems with partially prescribed eigenstructure. SIAM J. Matrix Anal. Appl., 25 (2004), 995-1020.

[5] M. Chu, M. Lin, B. Dong Semi-definite programming techniques for structured quadratic inverse eigenvalue problems. Numerical Algorithms, 53 (2010), no. 4, 419-437.

[6] G. Gasper Linearization of the product of Jacobi polynomials. I , II. Canad. J. Math., 22 (1970), 171-175, 582-593.

[7] R. Ibragimov, D. Pelinovsky. Incompressible viscous fluid flows in a thin spherical shell. J. Math. Fluid. Mech., 11 (2009), 60-90.

[8] W. Mlotkowski, R. Szwarc. Non-negative linearization for polynomials orthogonal with respect to discrete measures. Constr. Approx., 17 (2001), 413-429.

[9] V. Totik. Orthogonal Polynomials, Surveys in Approximation Theory., 1 (2005), 70-125. 